\title{
Detection of metallobetalactamase producing imipenem resistant acinetobacter species in intensive care unit patient in a Tertiary Care Centre
}

\author{
Vinoba S. ${ }^{1}$, Rashmi K. S., ${ }^{2, *}$ \\ ${ }^{1}$ Assistant Professor, ${ }^{2}$ Associate Professor, Dept. of Microbiology, ${ }^{1} \mathrm{P}$. K. Das Institute of Medical Sciences, \\ Vaniamkulam, ${ }^{2}$ Kempegowda Institute of Medical Sciences, Bengaluru, Karnataka, India
}

*Corresponding Author:

Email: vinobaps@gmail.com

\begin{abstract}
Introduction: Metallobetalactamase producing Acinetobacter species has been reported to be an important cause of nosocomial infection and is a critical therapeutic problem worldwide, especially in the intensive care unit.

Objectives: To determine the frequency of metallo- $\beta$-lactamases among imipenem-resistant Acinetobacter species and to compare different phenotypic methods.

Materials and Methods: 59 imipenem-resistant Acinetobacter species isolated from various clinical samples were tested for metallo- $\beta$-lactamase production using different phenotypic methods. Minimal Inhibitory Concentratrion (MIC) to meropenem was determined by E test.

Results: Of all the imipenem resistant isolates, $50.8 \%$ of Acinetobacter species were MBL producers. MIC of all those MBL producing isolates were $\geq 16 \mu \mathrm{g} / \mathrm{ml}$. Among the Acinetobacter species MBL production were detected in 30 isolates (50.8\%) by E test, 29 isolates $(49.2 \%)$ by CDT and in 15 isolates $(25.4 \%)$ by DDST and DPT. The sensitivity and specificity of CDT, DDST and DPT compared to E test was $98 \%, 48.1 \%, 48.1 \%$ and $100 \%$, respectively.

Conclusion: Prevalence of MBL producing Acinetobacter species is being increasingly reported in ICU patients. The MIC of all the MBL producing isolates for meropenem were $>16 \mu \mathrm{g} / \mathrm{ml}$ (Resistant). E test and CDT were more reliable for MBL detection. CDT was cost effective, easy to perform and interpretation also straightforward. MBL producing isolates were multidrug resistant making therapeutic choices limited. Continuous antibiotic surveillance, infection control practices and an effective antibiotic policy are required to address the problem of MBL - associated infections.
\end{abstract}

Keywords: Acinetobacter species, MBL detection.

\section{Introduction}

Acinetobacter species is the most common pathogen causing nosocomial infections., ${ }^{1,2}$ This organism is noted for their intrinsic resistance to antibiotics and for their ability to acquire genes encoding resistance determinants. ${ }^{3}$ Carbapenems are used as the last choice in treating serious infections caused by multidrug resistant gram negative bacilli. Unfortunately resistance to these antibiotics started emerging and has been reported worldwide. This resistance to carbapenem is due to decreased outer membrane permeability, increased efflux systems, alteration of penicillin binding proteins and carbapenem hydrolyzing enzymes carbapenemase. ${ }^{4}$ Among carbapenemases transferable MBL are the most feared because of their ability to hydrolyze all $\beta$ - lactams, including carbapenems, except monobactams. The capability of MBLs to disseminate and spread through bacterial population is facilitated by its gene being encoded on an intergron -borne mobile gene cassette. - $^{4-6}$ The rapid detection of $\mathrm{MBL}$ - producing GNB is necessary to aid infection control and to prevent dissemination. Also infection with MBLs result in higher mortality rates, probably related to less frequent institution of appropriate antimicrobial therapy. PCR though highly accurate and reliable, its accessibility is often limited to reference laboratories. MBL - activity is inhibited by chelating agents. Therefore several laboratory methods using metal chelators as EDTA have been described for the phenotypic detection of MBLs among clinical isolates., ${ }^{2,6}$

\section{Materials and Methods}

Source of Specimens: This study was conducted prospectively from January 2014 to June 2015 in the Department of Microbiology in a tertiary care centre. A total of 59 consecutive, non repetitive isolates of Acinetobacter species were included. They were isolated from various samples (Trachial aspirate, Pus, Sputum, Urine, Ascitic fluid, Pleural fluid) from intensive care unit patients.

Isolation and identification of organisms: Immediately after receiving the sample, the material was subjected to microscopic examination. After Gram staining specimens were inoculated onto MacConkey agar, Blood agar and Chocolate agar. Plates were incubated at $37^{\circ} \mathrm{C}$ for $18-24$ hours. Cultures yielding insignificant growth were excluded. The colonies were subjected to Gram's stain and other biochemical tests for identification.

Antibiotic susceptibility testing: Antibiotic susceptibility test was performed according to CLSI (Vol 32 No.2. M07 - A 9. Wayne PA: Jan 2012) guidelines. Susceptibility was tested against the following antibiotics:

1. Amikacin $(30 \mu \mathrm{g})$ 
2. Amoxyclav $(20 / 10 \mu \mathrm{g})$

3. Ampicillin $(10 \mu \mathrm{g})$

4. Cefepime $(30 \mu \mathrm{g})$

5. Cefuroxime $(30 \mu \mathrm{g})$

6. Cefoperazone $(75 \mu \mathrm{g})$

7. Ciprofloxacin $(5 \mu \mathrm{g})$

8. Cotrimoxazole $(1.25 / 23.75 \mu \mathrm{g})$

9. Gentamicin $(10 \mu \mathrm{g})$

10. Imipenem( $(10 \mu \mathrm{g})$

11. Levofloxacin $(5 \mu \mathrm{g})$

12. Meropenem $(10 \mu \mathrm{g})$

13. Piperacillin-Tazobactam $(100 / 10 \mu \mathrm{g})$

These discs were obtained from HiMedia Laboratories, Mumbai. The diameter of zone of inhibition was measured and interpreted according to CLSI (Vol 32 No.3. M100-S22.Wayne PA: Jan 2012) guidelines.

\section{MBL detection}

Imipenem- EDTA Combined disc test (CDT): This test was described by Yong et al. Here the test organisms were inoculated on to plates with Mueller Hinton agar (MHA) as per CLSI (Vol 32 No.2. M07 A 9. Wayne PA: Jan 2012) guidelines. Two $10 \mu \mathrm{g}$ Imipenem discs (Becton Dickinson) were placed on the plate, and appropriate amounts of $10 \mu \mathrm{L}$ of EDTA solution were added to one of them to obtain the desired concentration $(750 \mu \mathrm{g})$. The plates were incubated at $37^{\circ} \mathrm{C}$ for 24 hours. The inhibition zones of the Imipenem and Imipenem-EDTA discs were compared. The increase in inhibition zone with the Imipenem and EDTA disc $\geq 7 \mathrm{~mm}$ than the Imipenem disc alone, was considered as MBL positive. ${ }^{2}$

Imipenem- EDTA double disc synergy test (DDST): This test was described by Lee et al. Here the test organisms were inoculated on to MHA plates as per CLSI (Vol 32 No.2. M07 - A 9. Wayne PA: Jan 2012) guidelines. An Imipenem $(10 \mu \mathrm{g})$ disc was placed 20 $\mathrm{mm}$ center to center from a blank disc containing $10 \mu \mathrm{L}$ of $0.5 \mathrm{M}$ EDTA $(750 \mu \mathrm{g})$. Enhancement of the zone of inhibition in the area between Imipenem and the EDTA disc in comparison with the zone of inhibition on the far side of the drug was interpreted as a positive result. ${ }^{2}$

EDTA- disc potentiation test using ceftazidime, ceftizoxime, cefepime and cefotaxime (DPT): Here the test organisms were inoculated on to plates with MHA as described for the standard disc diffusion test. A filter paper blank disc is placed and the following discs Ceftazidime (30 $\mu \mathrm{g}), \quad$ Ceftizoxime $(30 \mu \mathrm{g})$, Cefepime $(30 \mu \mathrm{g})$, Cefotaxime $(30 \mu \mathrm{g})$ are placed $25 \mathrm{~mm}$ center to center from the blank disc. $10 \mu \mathrm{L}$ of $0.5 \mathrm{M}$
EDTA solution is added to the blank disc and the plate was incubated overnight at $35^{\circ} \mathrm{C}$ for enhancement of the zone of inhibition in the area between the EDTA disc and any one of the four Cephalosporin disc in comparison with the zone of inhibition on the far side of the drug was interpreted as a positive result. ${ }^{2}$

MBL E test

Broth microdilution methods usually detect carbapenem resistance. When performed properly, disc diffusion and agar gradient diffusion also are acceptable methods for carbapenem testing. An additional test method, such as agar gradient diffusion (i.e., E test), can be used to verify intermediate or resistant results.

Among carbapenems, imipenem degrades easily. Studies suggest meropenem may be more stable than imipenem. Hence compared to imipenem, meropenem is the best choice for calculating MIC of carbapenem in vitro. $^{7}$

Here in this study a unique phenotypic MBL detection E test strip were used, which is capable of detecting MBLs including strains with recently discovered NDM-1 resistant gene. This strip is coated with mixture of Meropenem + EDTA and Meropenem on a single strip in a concentration gradient manner. The upper half has Meropenem+EDTA (1to $64 \mu \mathrm{g} / \mathrm{ml}$ ) with highest concentration tapering downwards, whereas lower half is similarly coated with Meropenem (4 to $256 \mu \mathrm{g} / \mathrm{ml}$ ) in a concentration gradient in reverse direction. The $\mathrm{E}$ test was done according to manufacturer's instruction. MIC ratio of MRP/MRPEDTA of $>8 \log 2$ dilutions indicates MBL production and $<$ or $=$ to 8indicates MBL negative. ${ }^{8,9}$

\section{Results}

A total of 59 consecutive, non repetitive isolates of Acinetobacter species were studied in a tertiary care centre between January 2014 and June 2015 .

MIC of Meropenem measured by E test (Table 1).

$\mathrm{E}$ Test found to be most sensitive phenotypic method for detection of MBL production among imipenem resistant isolates (Table 2/Graph 1).

Compared to E Test, CDT 98\% sensitive and 100\% specific (Table 3)

73\% of Acinetobacter species from wound infection (other than post operative and diabetic foot) were MBL producers respectively (Table 4/Graph 2).

MBL producers were significantly resistant ( $\mathrm{P}$ value $<$ 0.05 ) to beta lactam drugs with beta lactamase inhibitors, fluoroquinolones and co-trimoxazole (Table 5/Graph 3).

Table 1: Detection of MIC of Meropenem by $E$ test

\begin{tabular}{|l|c|}
\hline MIC of Meropenem & E Test \\
\hline $0.5 \mu \mathrm{g} / \mathrm{ml}$ & 00 \\
\hline $1 \mu \mathrm{g} / \mathrm{ml}$ & 00 \\
\hline $2 \mu \mathrm{g} / \mathrm{ml}$ & 00 \\
\hline $4 \mu \mathrm{g} / \mathrm{ml}$ & 02 \\
\hline
\end{tabular}




\begin{tabular}{|l|l|}
\hline $8 \mu \mathrm{g} / \mathrm{ml}$ & 12 \\
\hline $16 \mu \mathrm{g} / \mathrm{ml}$ & 26 \\
\hline $32 \mu \mathrm{g} / \mathrm{ml}$ & 07 \\
\hline $64 \mu \mathrm{g} / \mathrm{ml}$ & 12 \\
\hline
\end{tabular}

Table 2: Detection of MBL production by different phenotypic methods

\begin{tabular}{|l|c|c|}
\hline \multicolumn{1}{|c|}{ Test } & MBL Positive & MBL Negative \\
\hline CDT & $29(49.2 \%)$ & $30(50.8 \%)$ \\
\hline DDST & $15(25.4 \%)$ & $44(74.6 \%)$ \\
\hline DPT & $15(25.4 \%)$ & $44(74.6 \%)$ \\
\hline E TEST & $30(50.8 \%)$ & $29(49.2 \%)$ \\
\hline
\end{tabular}

Table 3: Comparison of different phenotypic methods with $\mathrm{E}$ test in detection of MBL production.

\begin{tabular}{|l|l|l|l|l|}
\hline $\begin{array}{c}\text { Phenotypic } \\
\text { method }\end{array}$ & Sensitivity & Specificity & PPV & NPV \\
\hline CDT & $98 \%$ & $100 \%$ & $100 \%$ & $98 \%$ \\
\hline DDST & $48.1 \%$ & $100 \%$ & $100 \%$ & $64 \%$ \\
\hline DPT & $48.1 \%$ & $100 \%$ & $100 \%$ & $64 \%$ \\
\hline
\end{tabular}

Table 4: MBL production of Acinetobacter species from different clinical conditions

\begin{tabular}{|l|l|l|}
\hline \multicolumn{1}{|c|}{ Clinical condition } & $\begin{array}{c}\text { MBL } \\
\text { positive }\end{array}$ & $\begin{array}{c}\text { MBL } \\
\text { negative }\end{array}$ \\
\hline $\begin{array}{l}\text { Wound infection (Other than } \\
\text { postoperative and diabetic foot } \\
\text { infection) }\end{array}$ & $08(73 \%)$ & 03 \\
\hline Respiratory tract infections & $15(54 \%)$ & 13 \\
\hline Post operative wound infection & $04(44 \%)$ & 05 \\
\hline Diabetic foot & $02(33 \%)$ & 04 \\
\hline Blood stream infections & $01(50 \%)$ & 01 \\
\hline Urinary tract infections & $00(0 \%)$ & 02 \\
\hline Peritonitis & $00(0 \%)$ & 01 \\
\hline Total & 30 & 29 \\
\hline
\end{tabular}

Table 5: Antibiotic resistance pattern of MBL positive and MBL negative isolates of Acinetobacter species.

\begin{tabular}{|c|c|c|c|c|c|}
\hline \multirow[t]{2}{*}{ Antibiotic } & \multicolumn{2}{|c|}{$\begin{array}{l}\text { MBL Positive } \\
\quad(\mathbf{n}=\mathbf{3 0})\end{array}$} & \multicolumn{2}{|c|}{$\begin{array}{c}\text { MBL Negative } \\
\text { (29) }\end{array}$} & \multirow[t]{2}{*}{$\begin{array}{c}\text { P- } \\
\text { Value }\end{array}$} \\
\hline & No & $\%$ & No & $\%$ & \\
\hline $\mathrm{AK}$ & 19 & 63.33 & 16 & 55.17 & 0.54 \\
\hline AMC & 30 & 100 & 19 & 100 & - \\
\hline AMP & 30 & 100 & 27 & 93.10 & 0.14 \\
\hline CPM & 30 & 100 & 28 & 96.55 & 0.31 \\
\hline CXM & 30 & 100 & 29 & 100 & - \\
\hline CPZ & 30 & 100 & 26 & 89.66 & 0.07 \\
\hline CIP & 28 & 93.33 & 17 & 58.62 & $0.002^{*}$ \\
\hline COT & 28 & 93.33 & 19 & 65.51 & $0.008^{*}$ \\
\hline GEN & 20 & 66.67 & 18 & 62.07 & 0.71 \\
\hline LE & 0 & 00 & 02 & 6.90 & 0.14 \\
\hline PIT & 12 & 40 & 01 & 3.45 & $<0.001^{*}$ \\
\hline
\end{tabular}


Graph 1: Detection of MBL production by different phenotypic methods

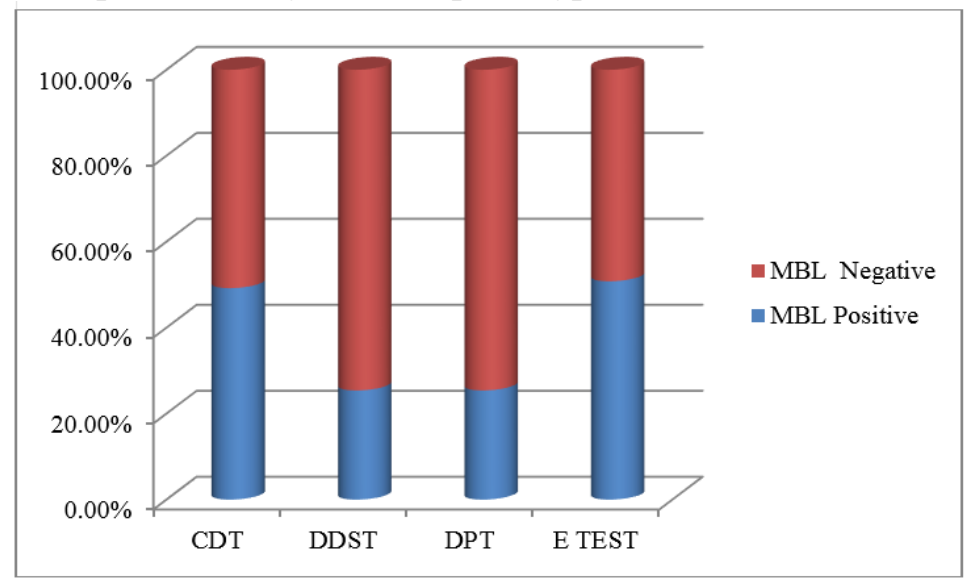

Graph 2: MBL production in Acinetobacter species from different clinical conditions

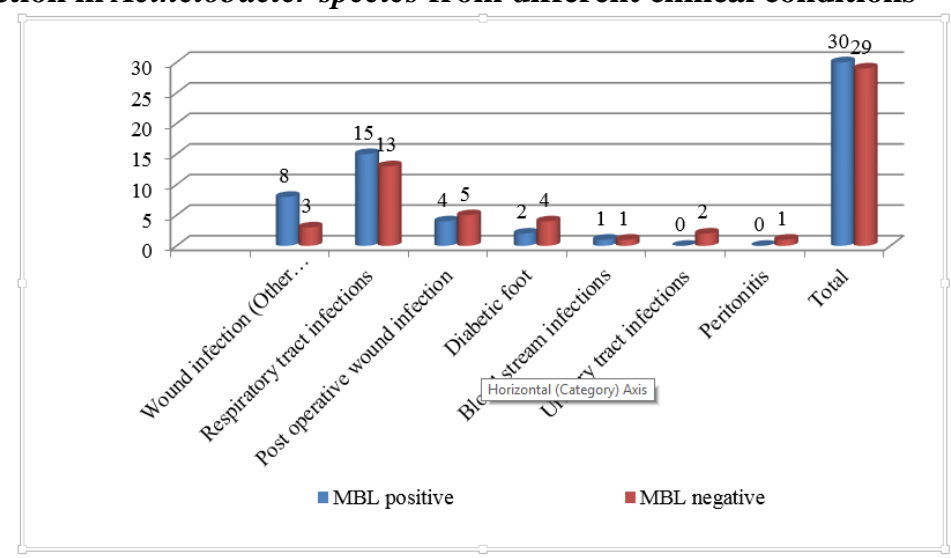

Graph 3: Antibiotic resistance pattern of MBL positive and MBL negative isolates of Acinetobacter species

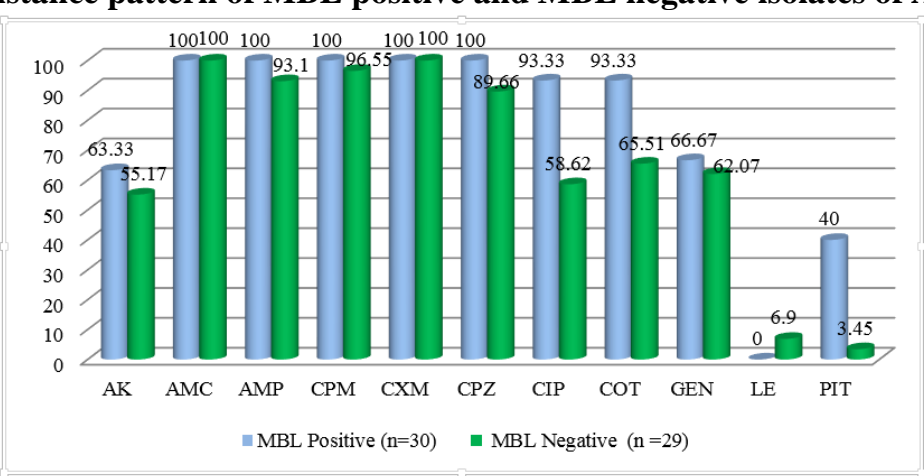

\section{Statistical Analysis}

Analysis was done by Epidata analysis software version 2.22.182.Discrete variables were expressed as percentages and proportions were compared using the Chi-square test. $\mathrm{P}$ value $<0.05$ was considered significant.

\section{Discussion}

The incidence of nosocomial infections in critically ill patients is much higher than in general ward patients ${ }^{[3]}$.Increasing incidence of serious infections in hospital intensive care units due to Methicillin resistant
Staphylococcus aureus, MDR Klebsiella spp, A. baumanni and $P$. aeruginosa are being reported worldwide. ${ }^{10,11}$ Most frequently encountered species are Acinetobacter species and Pseudomonas aeruginosa that are commonly associated with infections, such as bacteremia, urinary tract infection, meningitis, skin and soft tissue infections and pneumonia in ICU patients. $^{12,13}$ These organisms are noted for their intrinsic resistance to antibiotics and for their ability to acquire genes encoding resistance determinants. Because of frequent resistance to commonly used 
antibiotics, carbapenems have become important for managing these infections. ${ }^{14}$

Recently resistance to carbapenems has started emerging due to carbapenamases and expression of efflux pumps. Among carbapenemases, the genes responsible for MBL production may be chromosomally or plasmid mediated and hence pose a threat of spread of resistance by gene transfer among the Gram - Negative bacteria. ${ }^{4}$ Severe nosocomial infections due to MDR, MBL producing Acinetobacter species contribute to prolonged hospitalization, increased morbidity and mortality and of course, increased resource utilization. ${ }^{3}$

Correct identification of MBL producing organisms in due time is necessary not only for optimal patient management but also for immediate institution of appropriate infection control measures to prevent the spread of these organisms.

This study aims to identify an early, rapid and effective phenotypic method for identifying MBLs in ICU patients especially for Acinetobacter species which is being increasingly reported. In this study all isolates were Imipenem resistant and were subjected to E Test for measurement of their MIC to Meropenem. Imipenem degrades easily. Studies suggest that, Meropenem is slightly more active and stable than Imipenem against Gram - negative organisms. ${ }^{7}$ Hence, among carbapenems, meropenem was used in this study for measurement of MIC for all the isolates by E test. MIC values were interpreted based on the current CLSI breakpoints. $^{15}$

The MIC of 2 isolates were $\leq 4 \mu \mathrm{g} / \mathrm{ml}$ (sensitive), 12 isolates $\mathrm{MIC}$ were $8 \mu \mathrm{g} / \mathrm{ml}$ (intermediate susceptibility) and 45 isolates $\mathrm{MIC}$ were $\geq 16 \mu \mathrm{g} / \mathrm{ml}$ (Resistant) by E test.

Carbapenem resistance is usually multifactorial. Apart from carbapenem- hydrolyzing enzymes (MBLs), increased production of AmpC chromosome-encoded cephalosporinase, reduced outer membrane porinOprD expression, and associated factors are known to contribute to carbapenem resistance. Overexpression of the MexAB-OprM efflux system is known to affect meropenem efficacy but not that of imipenem. In addition, the MexCD-OprJ and MexXY-OprM efflux systems may also be involved in reduced susceptibility to meropenem. Whereas OprD inactivation alone is the source of intermediate susceptibility or resistance to imipenem. The mechanisms leading to meropenem resistance, involves overproduction of AmpC or over expression of the efflux pumps MexAB-OprM, MexXY-OprM, and MexCD-OprJ ${ }^{[16]}$. In the present study, the imipenem resistance in meropenem sensitive isolates could be due to OprDporin mutation. Hence all the isolates were tested for MBL production by different phenotypic tests. CDT, DDST and DPT were used for detecting MBL production in all the isolates and the sensitivity of CDT Test, DDST and DPT were
$98 \%, 48.1 \%$ and $48.1 \%$ and the specificity of CDT, DDST and DPT were $100 \%$ when compared to E Test.

There are conflicting reports regarding the performance of MBL E Test in the literature. It has been reported to be insensitive to detect carbapenem sensitive MBL carrying organisims. ${ }^{2}$ In our study, we screened only carbapenem resistant isolates with MBL E Test, which may have accounted for very high sensitivity of the test. We found that all isolates found to be MBL positive by CDT were also positive with the $\mathrm{E}$ Test and one isolate which were positive by $\mathrm{E}$ Test, were found to be MBL negative by CDT. These findings are consistent with the study done by Maria Omair et al. ${ }^{17}$

The CDT detected $48.2 \%$ of MBL producers among which were negative by the DDST and DPT. This may be because the sensitivity varies with the distance between the discs and the antibiotic discs chosen for the test. The major disadvantage of DDST and DPT were the subjective interpretation of result in some instances. The increase of $\geq 7 \mathrm{~mm}$ in inhibition zone with Imipenem plus EDTA disc than with Imipenem disc alone was considered as MBL positive by CDT. The CDT was found to be superior to DDST and DPT. Hence, in this study CDT was found to be one of the most sensitive techniques than DPT, DDST for detecting MBL. This findings are correlating with other studies. ${ }^{2,3}$ CDT is technically much simpler and inexpensive method compared to other phenotypic methods like E Test, DDST and DPT. The interpretation is straightforward. Hence, addition of one more imipenem disc with $0.5 \mathrm{M}$ EDTA along with regular imipenem disc while performing the disc diffusion method would also screen for $\mathrm{MBL}$ production in the laboratory.

\section{Conclusion}

MBL producing Acinetobacter species is being increasingly reported in ICU patients. The MIC of all the MBL producing isolates for meropenem were $>16 \mu \mathrm{g} / \mathrm{ml}$ (Resistant). Compared to DDST and DPT, E test and CDT were more reliable for MBL detection. Compared to E Test, CDT was cost effective, easy to perform and interpret. MBL producing isolates were multidrug resistant making therapeutic choices limited. Polymyxin B and Colistin are the next therapeutic options for carbapenem resistant isolates. Continuous antibiotic surveillance, infection control practices and an effective antibiotic policy are required to address the problem of MBL - associated infections. Molecular studies are necessary to evaluate the various MBL type.

\section{References}

1. Prashanth K, Badrinath S. Nosocomial infections due to Acinetobacter species: Clinical findings, Risk and Prognostic factors. Indian Journal Medical Microbiology, (2006)24 (1):39-44. 
2. Behera B, Mathur P, Kapil A, Sharma V. An evaluation of four different phenotypic techniques for detection of metallobetalactamase producing Pseudomonas aeruginosa. Indian Journal Of Medical Microbiology, (2008)26(3):233-7.

3. Varun Goel, Hogade SA, Karadesai SG. Prevalence of extended - spectrum beta -lactamases, Amp C beta lactamase,andmetallo - beta -lactamase producing Pseudomonas aeruginosa and Acinetobacter baumanniiin an intensive care unit in a tertiary care hospital.Journal of scientific society, vol 40/issue 1/January- April 2013.

4. Ami Varaiya, Nikhil Kulkarni,ManasiKulkarni,PallaviBhalekar ,Jyotsana Dogra. Incidence of Metallo beta lactamase producing Pseudomonas aeruginosa in ICU patients. Indian J Med Res 127,April 2008,pp 398-402.

5. Noyal MJC. ,Menezes GA, Harish BN, Sujatha S, ParijaSC.Simple screening tests for detection of carbapenemases in clinical isolates of nonfermentative gran - negative bacteria. Indian J Med Res 129, June 2009,pp 707-12.

6. Fam N, Diab M, Helmi H, El-DefrawyI.Phenotypic detection of metallo beta lactamases and extended spectrum beta lactamases among gram negative bacterial clinical isolates. Egyptian Journal of medical microbiology, octobar 2006

7. Laboratory detection of imipenem or meropenem resistance in Gram- Negative Organisms. Centers for disease control and prevention

8. Walsh TR, Bolmstrom A, Qwarnstrom A, Gales A. Evaluation of a new E test for detectingMetallo beta lactamases in routine clinical testing.7 May 2002.

9. Meropenem with and without EDTA Ezy MICTM Strip [Package insert] Mumbai: Himedia; 2012.

10. National Nosocomial Infections Surveillance (NNIS) system report, data summary from January 1992-April 2000, issued June 2000. Am J Infect Control 2000; 28:429.

11. Hidron AI, Edwards JR, Patel J, et al. NHSN annual update: antimicrobial-resistant pathogens associated with healthcare-associated infections: annual summary of data reported to the National Healthcare Safety Network at the Centers for Disease Control and Prevention, 2006-2007. Infect Control Hosp Epidemiol 2008;29:996.

12. Prashanth K, Badrinath $\mathrm{S}$. In Vitro susceptibility pattern of Acinetobacter species to commonly used cephalosporins,quinolones, and aminoglycosides. Indian Journal Of Medical Microbiology,(2004) 22 (2):97-103.

13. Amudhan SM, Sekar U, Arunagiri K, Sekar B. OXA Beta - lactamase mediated carbapenem resistance in Acinetobacter baumannii. Indian Journal Of Medical Microbiology,(2011) 29 (3):269-74.

14. Sinha S,Agarwal J, Srivastava S, Singh M. Analysis of carbapenem resistant Acinetobacter from a tertiary care setting in north India. Indian Journal Of Medical Microbiology,(2013)31(1):60-3.

15. Performance standards for antimicrobial susceptibility testing; Twenty - second informational supplement, Zone size and MIC interpretive criteria of A.baumannii andP.aeruginosa; Vol 32 No.3. M100-S22. Wayne PA: Jan 2012.

16. Rodriguez-Martinez JM, Poirel L, Nordmann P. Molecular epidemiology and mechanisms of carbapenem resistance in Pseudomonas aeruginosa.

Antimicrob.Agents Chemother.2009,53(11):4783.

17. Omair M, Usman J et al. Evaluation of combined disc method for the detection of metallo beta lactamase producing gram negative bacilli. Malaysian Journal of Microbiology,(2012),8(1):21-5.

How to cite this article: Vinoba $\mathrm{S}$, Rashmi KS. Detection of metallobetalactamase producing imipenem resistant acinetobacter species in intensive care unit patient in a Tertiary Care Centre. Indian J Microbiol Res 2018;5(1):132-137. 